\title{
37. SUMMARY AND INDEX TO PETROLOGIC AND GEOCHEMICAL STUDIES OF LEG 61 BASALTS ${ }^{1}$
}

\author{
R. Batiza, ${ }^{2}$ S. Shcheka,${ }^{3}$ H. Tokuyama,${ }^{4}$ K. Muehlenbachs,${ }^{5}$ T. L. Vallier, ${ }^{6}$ F. Lee-Wong, ${ }^{6}$ K. E. Seifert, ${ }^{7}$ \\ K. E. Windom, ${ }^{7}$ P. Book, ${ }^{7}$ and N. Fujii ${ }^{8}$
}

\section{INTRODUCTION}

Drilling during DSDP Leg 61 penetrated 1068.5 meters into the oceanic crust of the Nauru Basin. The bottom 504.5 meters of the hole penetrated a volcanicintrusive basalt complex which is interlayered with sediments in the upper portion (see Site Summary, this volume, for details of the Hole 462 stratigraphy). A total of about 320 meters of basalt was recovered in drilling a pilot hole (462: $33.7 \mathrm{~m}$ recovered) and a multiple re-entry hole (462A: $\sim 287 \mathrm{~m}$ recovered).

Over 300 sub-samples were examined by the shipboard petrologists and shore-based workers, and a great deal of petrographic, petrologic, and geochemical information has been collected for Leg 61 basalts. The purpose of this paper is to provide a brief review of these data and a summary of the findings. In addition, this paper gives electron microprobe mineral analyses and oxygen isotope data that are not contained elsewhere in this volume.

\section{STRATIGRAPHY}

The Nauru Basin basalt complex, as revealed by the drilling, consists of two major units. The upper unit (Cores 14-42 from Hole 462A and Cores 60-69 from Hole 462) consists of basalt sills with intercalated volcanogenic sediments. The sills vary greatly in thickness from less than one meter to over 50 meters, but are all basaltic in composition. They intrude sediments which, on the basis of included fossils, range from Cenomanian(?) to Hauterivian-Barremian. This range is consistent with an ${ }^{40} \mathrm{Ar} /{ }^{39} \mathrm{Ar}$ age determination of $110 \pm 3 \mathrm{~m} . \mathrm{y}$. on a sample from the upper sill complex (Ozima et al., this volume).

The lower unit of the igneous complex (Cores 44 to 92 from Hole 462A) consists of basalt flows of variable thickness, and is separated from the upper sill unit by about 10 meters of Hauterivian-Barremian sediment. $\mathrm{An}{ }^{40} \mathrm{Ar} /{ }^{39} \mathrm{Ar}$ age determination of a basalt sample from the lower unit gave a very poor isochron and a total fu-

\footnotetext{
1 Initial Reports of the Deep Sea Drilling Project, Volume 61.

2 Department of Earth and Planetary Sciences, Washington University, St. Louis, Missouri.

${ }^{3}$ Far East Institute of Geology, U.S.S.R. Academy of Sciences, Vladivostok, U.S.S.R.

4 Ocean Research Institute, University of Tokyo, Nakano, Tokyo 164 Japan.

5 Department of Geology, University of Alberta, Edmonton, Canada T6G, 2E3.

6 U.S. Geological Survey, Menlo Park, California.

7 Department of Earth Sciences, lowa State University, Ames, lowa.

8 Department of Earth Sciences, Kobe University, Kobe 657, Japan.
}

sion age of 131 m.y., the significance of which is difficult to interpret (Ozima et al., this volume).

\section{LITHOLOGY}

Macroscopic descriptions of the core samples are contained in the Site Summary (this volume). The sills of the upper unit have altered glassy margins, and the grain size of the basalt increases toward the center of the sills. The sills show clear contact-metamorphic effects in the intruded sediments (see Shcheka, this volume). One very thick sill contains late-magmatic granophyric patches consisting of intergrown quartz and potassium feldspar with two kinds of amphibole. Most of the sills contain veins and patches of secondary minerals, and are altered to variable degrees. Each sill appears to be a single intrusion and there is little evidence for multiple intrusions. (See Tokuyama and Batiza, this volume, for further discussion.) Uncommon grain-size inversion observed in the sills could result from multiple intrusion, but can also be attributed to local mass movements within a single cooling unit or to complex isotherm distribution within cooling sills.

The flows of the lower unit consist of upper glassy parts and thick, mostly holocrystalline lower parts. The glassy tops consist of multiple, thin, tabular, and contorted units, each consisting of upper and lower glassy margins, up to $5 \mathrm{~cm}$ thick, separated by fine-grained, more crystalline basalt. These grade downward into crystalline basalt units as thick as $\mathbf{3 0}$ or $\mathbf{4 0}$ meters. The thickness of the glassy portion of the units is usually a few centimeters, but several thick sequences (up to 30 $\mathrm{m}$ ) of glassy material (with poor recovery) were cored.

These units are interpreted as basalt sheet flows similar to those described by Ballard et al. (1979). The glassy portions are thought to represent flow lobes on the top surface of a rapidly moving flow, and the great local thickness of packets of thin glassy units may indicate close proximity to a vent. The thicker, non-glassy portions probably represent the more massive flow interiors. Fresh glass is preserved in some of these units, but in general the basalt is veined and replaced with secondary minerals. No unambiguous pillow lava was cored in the igneous complex.

\section{PETROGRAPHY}

More than 200 thin sections of the Nauru Basin igneous complex were examined on board during Leg 61 . Petrographic descriptions are given in the Site Summary 
(this volume), and mineralogic and textural data are summarized in Tables I-2 and I-5 of the same report. Plates 1 to 4 of this report show representative primary and replacement textures of Leg 61 basalts.

The holocrystalline basalts all contain plagioclase, augite, $\mathrm{Fe}-\mathrm{Ti}$ oxides, and secondary minerals. Many contain pseudomorphs of euhedral olivine with small inclusions of spinel, but some of the flows contain fresh olivine and spinel within fresh glass. Textures in the basalts very widely; this probably reflects crystallization and cooling at various cooling rates and degrees of undercooling. The textures range from glassy to variolitic and hyalopilitic and holocrystalline. Most holocrystalline basalts are patchy, and contain angular, finegrained or glassy domains (see Plates 1 and 4).

All the basalts are either aphyric or only sparsely phyric with less than $10 \%$, and generally less than $3 \%$, phenocrysts. The observed crystallization sequences of the basalts are discussed by Tokuyama and Batiza (this volume). Plagioclase or spinel and olivine appear to be the liquidus phases of Leg 61 basalts, and are followed quickly by pyroxene. In rare cases, pyroxene appears to be the liquidus phase.

\section{CHEMICAL STUDIES}

Table 1 gives a summary of the types of analyses performed on Leg 61 basalts. Onboard X-ray fluorescence (XRF) analyses of major elements in 146 samples has been supplemented by 42 additional wet-chemical majorelement analyses and 12 partial atomic-absorption analyses. Trace-element analyses have been completed for about 100 samples, and over 150 mineral analyses have been carried out by electron microprobe. Results of these various analyses are briefly summarized below.

\section{Mineral Chemistry}

\section{Olivine}

Eighteen analyses of olivine from five samples are presented by Shcheka (this volume) and Tokuyama and Batiza (this volume). The fresh olivines analyzed have a narrow compositional range of $\mathrm{Fo}_{83}$ to $\mathrm{Fo}_{84}$ and are not zoned. Fresh olivine is rarely preserved in Leg 61 basalts, and is usually replaced by secondary phases; thus, this narrow compositional range probably does not reflect the true primary variation of olivine compositions.

\section{Spinel}

Spinel occurs as small octahedral crystals in fresh glass or within olivine crystals in the section in flows. Twenty-four spinel analyses on eight samples are given by Shcheka (this volume) and Tokuyama and Batiza (this volume). In these samples, the $\mathrm{Cr} /(\mathrm{Cr}+\mathrm{Al})$ ratio varies from 0.54 to $0.73 ; \mathrm{Mg} /(\mathrm{Mg}+\mathrm{Fe})$ ranges from 0.49 to 0.54 . The samples are thus more chrome- and iron-rich than spinels generally occurring in mid-ocean ridge basalts (MORB). In addition, they apparently contain more $\mathrm{Fe}^{+3}$ than MORB spinels. It is worth noting that coexisting spinels, olivine, and glass all have lower
Table 1. Samples and analyses completed for Leg 61 basalts.

\begin{tabular}{|c|c|c|c|}
\hline $\begin{array}{l}\text { Core-Sec., } \\
\text { Interval (cm) }\end{array}$ & $\begin{array}{l}\text { Type of Analysis } \\
\text { (see notes) }\end{array}$ & $\begin{array}{l}\text { Core-Sec., } \\
\text { Interval (cm) }\end{array}$ & $\begin{array}{l}\text { Type of Analysis } \\
\text { (see notes) }\end{array}$ \\
\hline Hole 462 & & $21-1,140-143$ & $14,19,26,27$ \\
\hline 21-1, & 1,2 & $21-1,146-149$ & 17 \\
\hline Hole 462 & & $21-2,26-29$ & 17 \\
\hline $60-1,90-92$ & 16 & $22-1,95-100$ & $\begin{array}{l}17 \\
17\end{array}$ \\
\hline $60-2,139-142$ & $\begin{array}{l}16 \\
16\end{array}$ & $\begin{array}{l}22-1,137-139 \\
22-2,111-115\end{array}$ & $\begin{array}{l}17 \\
17\end{array}$ \\
\hline $61-2,53-56$ & 16 & $22-2,120-124$ & 14,19 \\
\hline $62-1,79-81$ & 16 & $22-3,12-14$ & 17 \\
\hline $62-1,127-129$ & 16 & $22-4,83-85$ & 17 \\
\hline $63-1,132-136$ & 16 & $22-5,4-6$ & 17 \\
\hline $63-2,79-83$ & 16 & $22-5,77-79$ & 17 \\
\hline $63-3,119-122$ & 16 & $23-1,25-29$ & 17 \\
\hline $64-1,4-6$ & 16 & $23-2,44-47$ & 17 \\
\hline $64-1,8-11$ & 14,22 & $23-2,69-71$ & 17 \\
\hline $64-3,79-81$ & 16 & $23-2,95-98$ & 17 \\
\hline $64-4,74-76$ & 16 & $23-3,75-77$ & 17 \\
\hline $65-1,27-30$ & 16 & $23-4,48-50$ & 17 \\
\hline $65-1,120-124$ & $6,9,12,13$ & $24-1,8-10$ & 17 \\
\hline $65-2,23-26$ & 16 & $24-1,27-31$ & 17 \\
\hline $66-1,19-21$ & 16 & $24-3,81-85$ & 26,27 \\
\hline $66-1,23-25$ & 14,22 & $24-4,51-54$ & 17 \\
\hline $66-3,93-97$ & $9,12,13$ & $25-1,39-42$ & 17 \\
\hline $66-4,12-14$ & 16 & $25-1,99-102$ & 17 \\
\hline $66-4,16-20$ & 14 & B-1, 23-26 & 17 \\
\hline $66-4,97-101$ & $9,12,13$ & B- $1,40-43$ & 17 \\
\hline $66-5,42-47$ & 14 & $27-1,31-33$ & 17 \\
\hline $66-5,52-55$ & 12,13 & $27-1,51-56$ & 21 \\
\hline $67-1,63-67$ & $9,12,13$ & $27-1,94-97$ & 14,19 \\
\hline $67-2,90-94$ & 16 & $27-1,97-100$ & 17 \\
\hline $68-1,67-71$ & 14 & $27-2,71-76$ & 12 \\
\hline $68-1,73-75$ & 16 & $27-2,78-80$ & 17 \\
\hline $68-1,81-85$ & $9,12,13$ & $27-2,125-128$ & 17 \\
\hline $68-2,34-36$ & 14 & $27-3,71-74$ & 17 \\
\hline $68-2,37-39$ & 16 & $28-1,22-24$ & 15 \\
\hline $68-2,49-51$ & 16 & $28-1,96-98$ & 17 \\
\hline $68-2,105-110$ & $9,12,13$ & $28-2,28-30$ & 17 \\
\hline $68-3,55-60$ & $9,12,13$ & $28-2,73-75$ & 17 \\
\hline $69-1,17-21$ & $4,5,6,7,9,12,13$ & $28-2,81-83$ & 12 \\
\hline $69-1,20-24$ & $14,18 a$ & $28-3,1-2$ & 14,19 \\
\hline $69-1,36-38$ & 16 & $28-3,8-10$ & 17 \\
\hline $69-2,85-89$ & $7,9,12,13$ & $28-3,19-22$ & 21 \\
\hline Hole 462A & & $28-3,100-103$ & 21 \\
\hline & 17 & $28-3,134-136$ & 17 \\
\hline $\begin{array}{l}14-2,4-7 \\
14-2,29-32\end{array}$ & $\begin{array}{l}17 \\
17\end{array}$ & $28-4,68-70$ & 17 \\
\hline $\begin{array}{l}14-2,29-32 \\
14-2,48-50\end{array}$ & $\begin{array}{l}17 \\
21\end{array}$ & $28-5,26-28$ & 17 \\
\hline $14-2,48-50$ & & $29-1,6-9$ & 17 \\
\hline $14-2,68-70$ & 21 & $29-3,78-82$ & 14,19 \\
\hline $14-2,82-87$ & 19 & $29-5$ & 21 \\
\hline $15-1,66-68$ & 19 & $29-6,86-88$ & 12 \\
\hline $15-1,117-120$ & 17 & $29-6,97-100$ & 17 \\
\hline $15-2,41-48^{\mathrm{a}}$ & 21 & $30-1,48-50$ & 17 \\
\hline $15-2,41-48^{b}$ & 21 & $30-1,98-100$ & 12 \\
\hline $16-1,97-99$ & 21 & $30-3,14-17$ & 12 \\
\hline $16-1,120-122$ & 21 & $30-3,21-23$ & 17 \\
\hline $16-1,129-131$ & 17 & $30-4,82-84$ & 15 \\
\hline $17-1,17-21$ & 21 & $30-6,85-87$ & 15 \\
\hline $17-1,98-100$ & 17 & $31-1,48-50$ & 17 \\
\hline $17-1,117-119$ & 21 & $32-1,24-28$ & 14,19 \\
\hline $17-2,36-38$ & 17 & $32-1,44-48$ & 17 \\
\hline $17-2,84-86$ & $14,19,22$ & $33-2,10-12$ & 17 \\
\hline $18-1,128-130$ & $17=0.2$ & $36, \mathrm{CC}, 21-23$ & 17 \\
\hline $18-2,48-50$ & 17 & $38-1,53-55$ & 17 \\
\hline $19-1,10-12$ & 17 & $38-2,18-20$ & 17 \\
\hline $19-1,49-51$ & 21 & $38-2,43-46$ & 4,5 \\
\hline $19-1,52-54$ & 17 & $38-2,53-56$ & 14,19 \\
\hline $19-2,7-10$ & 21 & $39-1,59-61$ & 17 \\
\hline $19-2,90-92$ & 17 & $39-2,97-99$ & 17 \\
\hline $20-1,72-74$ & 17 & $39-2,100-104$ & 14,19 \\
\hline $20-1,115-121$ & 14 & $39-3,76-78$ & 17 \\
\hline $20-1,116-121$ & 18,22 & $39-3,142-144$ & 17 \\
\hline $20-1,144-146$ & 17 & $39-4,4-6$ & 17 \\
\hline $20-2,93-95$ & 17 & $39-4,67-70$ & 17 \\
\hline $21-1,40-42$ & 17 & $39-5,98-100$ & 17 \\
\hline $21-1,93-96$ & 17 & $40-1,56-59$ & $18 \mathrm{a}, 18 \mathrm{~b}$ \\
\hline $21-1,123-125$ & 17 & $40-1,86-90$ & 17 \\
\hline
\end{tabular}


Table 1. (Continued).

\begin{tabular}{|c|c|c|c|}
\hline $\begin{array}{l}\text { Core-Sec., } \\
\text { Interval (cm) }\end{array}$ & $\begin{array}{l}\text { Type of Analysis } \\
\text { (see notes) }\end{array}$ & $\begin{array}{l}\text { Core-Sec., } \\
\text { Interval (cm) }\end{array}$ & $\begin{array}{c}\text { Type of Analysis } \\
\text { (see notes) }\end{array}$ \\
\hline Hole 462A & & $61-2,26-29$ & 21 \\
\hline $40-2,23-25$ & 17 & $61-2,115-118$ & 17 \\
\hline $40-2,41-43$ & 17 & $62-2,10-12$ & 17 \\
\hline $41-1,10-12$ & 17 & $62-2,128-132$ & $23,24,25,27$ \\
\hline $41-1,130-133$ & 17 & $62-3,119-122$ & 21 \\
\hline $41-2,110-112$ & 17 & $63-1,15-18$ & 21 \\
\hline $41-4,80-82$ & 17 & $63-1,19-22$ & $4,5,10$ \\
\hline $41-4,86-90$ & 14,19 & $63-1,36-38$ & 17 \\
\hline $41-5,78-80$ & 17 & $64-4,30-32$ & 17 \\
\hline $41-6,100-102$ & 17 & $65-1,1-6$ & 9,13 \\
\hline $41-7,96-99$ & 17 & $65-1,59-61$ & $14,19,22,26,27$ \\
\hline $41-7,106-109$ & 17 & $65-1,78-82$ & 21 \\
\hline $44-1,66-71$ & 14,19 & $65-2,60-62$ & 17 \\
\hline $44-1,67-70$ & 14,21 & $65-3,18-20$ & 12,13 \\
\hline $44-1,128-130$ & 17 & $65-3,67-70$ & 21 \\
\hline $44-2,7-9$ & 17 & $66-1,143-147$ & $6,7,12,13$ \\
\hline $44-2,12-16$ & $3,5,10,11$ & $66-3,143-148$ & $6,9,12,13,17$ \\
\hline $44-2,53-59$ & 17 & $66-5,145-150$ & $9,12,13$ \\
\hline $44-2,82-87$ & 14 & $66-7,33-35$ & 17 \\
\hline $44-2,106-109$ & 17 & $67-4,107-112$ & $9,12,13$ \\
\hline $45-1,7-8$ & 17 & $67-6,124-126$ & 17 \\
\hline $45-1,28-30$ & 21 & $67-7,21-25$ & $6,9,12,13$ \\
\hline $45-2,14-17$ & 17 & $68-2,72-74$ & $9,12,13$ \\
\hline $45-3,50-52$ & 17 & $68-2,104-106$ & $9,12,13$ \\
\hline $45-5,92-94$ & 17 & $68-3,119-122$ & 14,19 \\
\hline $46-2,61-63$ & 17 & $68-4,116-118$ & 17 \\
\hline $46-2,72-74$ & 17 & $69-1,80-84$ & 14,19 \\
\hline $46-2,122-124$ & 17 & $69-2,68-72$ & $14,19,23,26,27$ \\
\hline $46-6,118-120$ & 17 & $70-1,2-4$ & 17 \\
\hline $47-1,7-9$ & 21 & $70-3,64-66$ & 21 \\
\hline $47-1,88-89$ & 17 & $71-1,71-72$ & 17 \\
\hline $47-1,95-98$ & $3,5,10,11$ & $72-2,53-58$ & 17 \\
\hline $47-2,90-94$ & 14,19 & $72-2,63-66$ & 14,19 \\
\hline $47-2,98-100$ & 17 & $72-2,124-126$ & 17 \\
\hline $47-3,14-17$ & $23,24,25,27$ & $72-3,21-23$ & $14,19,22$ \\
\hline $47-3,74-77$ & $10,11-12,13$ & $73-1,28-30$ & 21 \\
\hline $48-1,100-102$ & 17 & $74-1,78-80$ & 21 \\
\hline $48-1,125-128$ & 21 & $74-4,25-31$ & $14,19,22$ \\
\hline $48-2,106-109$ & $23,24,25,27$ & $74-4,46-50$ & 14,19 \\
\hline $48-4,46-48$ & $4,5,10$ & $74-4,72-74$ & 17 \\
\hline $48-4,52-54$ & 17 & $75-2,25-28$ & 15 \\
\hline $48-4,72-74$ & 17 & $75-3,112-114$ & 12,13 \\
\hline $48-4,99-101$ & 21 & $75-5,115-118$ & 15 \\
\hline $48-4,101-104$ & 21 & $76-1,41-43$ & 20 \\
\hline $49-1,55-58$ & 17 & $76-1,60-62$ & 21 \\
\hline $49-1,83-85$ & 17 & $77-1,25-28$ & 15 \\
\hline $49-3,65-68$ & 14,19 & $77-1,136-138$ & 21 \\
\hline $49-3,70-72$ & 17 & $77-3,16-19$ & 15 \\
\hline $49-4,41-43$ & 17 & $78-1,61-63$ & 21 \\
\hline $50-2,60-63$ & 26,27 & $78-3,40-43$ & 12,13 \\
\hline $50-2,71-73$ & 17 & $79-1,111-113$ & 21 \\
\hline $50-3,11-14$ & 14,19 & $79-3,79-81$ & 20 \\
\hline $50-3,15-17$ & 17 & $79-5,38-41$ & 15 \\
\hline $50-3,109-112$ & 21 & $79-5,127-130$ & 20 \\
\hline $50-3,130-134$ & $14,19,22$ & $79-5,144-146$ & 12,13 \\
\hline $50-6,109-111$ & 17 & $80-2,91-93$ & 13 \\
\hline $51-4,17-22$ & 14,19 & $80-3,58-61$ & 20 \\
\hline $51-4,27-30$ & $12,13,17$ & $80-3,145-148$ & 20 \\
\hline $52-4,49-51$ & 17 & $80-4,36-39$ & 20 \\
\hline $56-1,61-64$ & 14,19 & $80-5,91-93$ & 12 \\
\hline $56-1,65-68$ & 17 & $81-1,15-16$ & 7 \\
\hline $56-1,131-134$ & $14,18 \mathrm{a}, 18 \mathrm{~b}, 19$ & $81-1,27-29$ & 21 \\
\hline $56-1,145-147$ & 17 & $81-2,15-16$ & 12,13 \\
\hline $56-2,95-98$ & 21 & $81-2,73-76$ & 15 \\
\hline $58-1,1-2$ & 21 & $81-3,63-65$ & 20 \\
\hline $58-3,123-125$ & 21 & $84-3,22-25$ & 15 \\
\hline $58-4,99-101$ & 17 & $84-4,7-10$ & 15 \\
\hline $59-3,132-134$ & 17 & $84-4,49-51$ & 21 \\
\hline $59-6,131-133$ & 17 & $84-4,56-58$ & 20 \\
\hline $60-3,99-101$ & 17 & $84-6,20-23$ & 15 \\
\hline $60-3,100-102$ & 14,19 & $85-2,36-39$ & 15 \\
\hline $61-1,1-6$ & 12 & $85-4,78-80$ & $8,12,13$ \\
\hline $61-1,21-24$ & 21 & $87-1,70-72$ & 12,13 \\
\hline $61-1,30-32$ & 17 & $87-1,84-89$ & 15 \\
\hline $61-1,146-149$ & 21 & $87-1,105-107$ & 19,20 \\
\hline
\end{tabular}

Table 1. (Continued).

\begin{tabular}{ll|ll}
\hline $\begin{array}{c}\text { Core-Sec., } \\
\text { Interval (cm) }\end{array}$ & $\begin{array}{c}\text { Type of Analysis } \\
\text { (see notes) }\end{array}$ & $\begin{array}{c}\text { Core-Sec., } \\
\text { Interval (cm) }\end{array}$ & $\begin{array}{c}\text { Type of Analysis } \\
\text { (see notes) }\end{array}$ \\
\hline Hole 462A & & $89-3,122-125$ & 15 \\
$88-2,67-70$ & 15 & $89-3,125-127$ & 19,20 \\
$88-2,74-75$ & 12,13 & $90-1,24-26$ & 21 \\
$88-2,95-97$ & 19,20 & $90-1,141-143$ & 21 \\
$88-2,100-102$ & 19,20 & $90-2,18-20$ & 21 \\
$88-3,9-11$ & 19,20 & $90-2,37-39$ & 21 \\
$89-2,38-40$ & 21 & $90-3,88-90$ & 12,13 \\
$89-3,58-60$ & 21 & $90-4,63-65$ & 19,20 \\
$89-3,60-66$ & 21 & $90-4,92-95$ & 15 \\
\hline
\end{tabular}

Notes:

1. Probe compositions of glass shards in sediment, Shcheka (this volume, table 1).

2. Probe compositions of mineral grains in sediment, Shcheka (this volume, table 2).

3. Olivine probe analyses, Shcheka (this volume, table 3).

4. Pyroxene probe analyses, Shcheka (this volume, table 4).

5. Feldspar probe analyses, Shcheka (this volume, table 5).

6. Order-disorder relations of plagioclase, Shcheka (this volume, table 6).

7. Magnetite and ilmenite probe analyses, Shcheka (this volume, table 7).

8. Probe analyses of silica-bearing magnetite, Shcheka (this volume, table 8).

9. Trace-element contents of magnetite, Shcheka (this volume, table 9).

10. Probe analyses of spinels, Shcheka (this volume, table 11).

11. Probe analyses of glasses, Shcheka (this volume, table 13).

12. Wet chemical whole-rock major-element analyses, Shcheka (this volume, table 14).

13. Whole-rock trace-element abundances: $\mathrm{Cr}, \mathrm{V}, \mathrm{Ni}, \mathrm{Co}, \mathrm{Zr}, \mathrm{Sn}, \mathrm{Rb}, \mathrm{Sr}$. Shcheka (this volume, table 16).

14. Whole-rock trace-element abundances: $\mathrm{La}, \mathrm{Ce}, \mathrm{Sm}, \mathrm{Eu}, \mathrm{Tb}, \mathrm{Yb}, \mathrm{Lu}$, $\mathrm{FeO}, \mathrm{Sc}, \mathrm{Cr}, \mathrm{Co}, \mathrm{Ni}, \mathrm{Zn}, \mathrm{Na}_{2} \mathrm{O}, \mathrm{Sr}, \mathrm{Hf}, \mathrm{Th}, \mathrm{Zr}, \mathrm{Rb}$. Batiza (this volume, table 1).

15. Whole-rock analyses for $\mathrm{Al}, \mathrm{Ba}, \mathrm{Ca}, \mathrm{Fe}, \mathrm{K}, \mathrm{Li}, \mathrm{Mg}, \mathrm{Mn}, \mathrm{Na}, \mathrm{P}, \mathrm{Sc}$, $\mathrm{Sr}, \mathrm{Ti}, \mathrm{V}, \mathrm{Y}, \mathrm{Rb},{ }^{87} \mathrm{Sr} / 86 \mathrm{Sr}$. Fujii et al. (this volume, table 1 ).

16. Onboard $\mathrm{X}$-ray fluorescence analyses for major oxides except $\mathrm{Na}_{2} \mathrm{O}$ : Site Summary (this volume). Also in visual core descriptions.

17. Onboard X-ray fluorescence analyses for major oxides except $\mathrm{Na}_{2} \mathrm{O}$, $\mathrm{MnO}$, and $\mathrm{P}_{2} \mathrm{O}_{5}$ : Site Summary (this volume). Also in visual core descriptions.

18a. Plagioclase probe analyses (F. Lee-Wong, analyst), this chapter.

$18 \mathrm{~b}$. Pyroxene probe analyses (F. Lee-Wong, analyst), this chapter.

19. Oxygen isotope ratios (K. Muehlenbachs, analyst), this chapter.

20. Whole-rock major- and trace-element abundances: $\mathrm{SiO}_{2}, \mathrm{Al}_{2} \mathrm{O}_{3}$, $\mathrm{FeO}, \mathrm{MgO}, \mathrm{CaO}, \mathrm{Na}_{2} \mathrm{O}, \mathrm{K}_{2} \mathrm{O}, \mathrm{MnO}, \mathrm{La}, \mathrm{Ce}, \mathrm{Sm}, \mathrm{Eu}, \mathrm{Tb}, \mathrm{Yb}, \mathrm{Lu}$ $\mathrm{Sc}, \mathrm{Cr}, \mathrm{Co}, \mathrm{Sr}, \mathrm{Jf}, \mathrm{Th}$, and Ta. Seifert (this volume, table 1).

21. X-ray and optical determinations of vein minerals, Windom and Book (this volume, table 1).

22. Wet chemical whole-rock major-element analyses, Tokuyama and Batiza (this volume, table 1).

23. Probe analyses of glasses, Tokuyama and Batiza (this volume, table 2).

24. Probe analyses of spinels, Tokuyama and Batiza (this volume, table 3).

25. Olivine probe analyses, Tokuyama and Batiza (this volume, table 4).

26. Pyroxene probe analyses, Tokuyama and Batiza (this volume, table 5).

27. Feldspar probe analyses, Tokuyama and Batiza (this volume, table 6).

$\mathrm{Mg} /(\mathrm{Mg}+\mathrm{Fe})$ ratios than those of comparable occurrences in MORB. Further, the spinels of Leg 61 lavas apparently coexist with more magnesian olivine than would be obtained by extrapolation of spinel compositional trends for MORB, but with much more iron olivine than spinel for the Stillwater complex (Tokuyama and Batiza, this volume). Application of the revised olivine-spinel geothermometer (Roeder et al., 1979) to data for coexisting olivine and spinel of Sample $462 \mathrm{~A}-47-3,14-17 \mathrm{~cm}$ yields a temperature of about $1150^{\circ} \mathrm{C}$, but the variation of mineral compositions 
within the sample results in an uncertainty of about $100^{\circ} \mathrm{C}$.

\section{Pyroxene}

Thirty-seven analyses of pyroxene from nine samples of Leg 61 basalt are given by Shcheka (this volume) and Tokuyama and Batiza (this volume). Nine additional analyses from two samples are given in Table 2 of this paper. The compositions show little variation in $\mathrm{Mg} /(\mathrm{Mg}+\mathrm{Fe})$, but both Ca-rich augites and $\mathrm{Ca}$-poor pigeonite are present in the basalts; pigeonite is restricted to microphenocrysts in the flows. In general, the clinopyroxenes of the sill complex are more iron-rich than those of the flows. Most pyroxenes are at least slightly zoned to more iron-rich compositions at the rims, and some exhibit sector zoning. Pyroxenes have low $\mathrm{Ti}, \mathrm{Na}$, and tetrahedral $\mathrm{Al}$ contents, and in general are very similar chemically to pyroxenes from drilled and dredged MORB samples (Tokuyama and Batiza, this volume; Shcheka, this volume).

\section{Feldspar}

Shcheka (this volume) and Tokuyama and Batiza (this volume) give 33 feldspar analyses from 14 samples. Fourteen additional analyses from three samples are given in Table 3 of this paper. The cores of plagioclase phenocrysts in the upper sills are usually about $\mathrm{An}_{70}$, whereas cores of phenocrysts in the lower flows are generally more calcic $\left(\mathrm{An}_{80}\right)$. This is consistent with the compositions of mafic minerals and whole-rock majorand trace-element chemistry of the sills and flows. As is usual, groundmass plagioclase laths are 10 to $15 \mathrm{~mole} \%$ richer in albite component than are phenocrysts in the same sample. Phenocrysts commonly exhibit normal zonation, and compositions from core to rim may vary by up to 15 mole $\%$ anorthite. Granophyric patches of the thick sill in the upper sill complex contain orthoclase that is highly disordered (Shcheka, this volume). Plagioclase order-disorder studies (Shcheka, this volume) indicate that, in general, plagioclase of Leg 61 basalts exhibits partial $\mathrm{Al}-\mathrm{Si}$ ordering.

\section{Fe and Ti Oxides}

Shcheka (this volume) presents a special study of the Fe-Ti oxides. In addition to magmatic oxides, which include the phases magnetite, ilmenite, and titanomagnetite, he describes rare Si-bearing magnetite which occurs as a secondary mineral in veins of some of the basalts. Shcheka presents major-and trace-element data for both magmatic and secondary $\mathrm{Fe}-\mathrm{Ti}$ oxides. He concludes that although the magmatic $\mathrm{Fe}-\mathrm{Ti}$ oxides are most similar chemically to ones previously described for MORB samples, the Nauru Basin magmatic opaques also have chemical affinities with continental flood basalts.

\section{Alteration of Leg 61 Basalts}

Windom and Book (this volume) undertook studies of vein material in Leg 61 basalts that, together with petrographic observations and oxygen isotope data (Table 4, this paper), give an integrated picture of the alteration history of Leg 61 basalts. By stratigraphic criteria, the earliest alteration event was that affecting the lower section of flows. It was characterized by the stabilization of zeolite, smectites, pyrite, calcite and minor quartz, talc(?), and apophyllite, and was probably similar to the non-oxidative alteration of normal MORB by hydrothermal circulation which occurs

Table 2. Pyroxene analyses.

\begin{tabular}{|c|c|c|c|c|c|c|c|c|c|}
\hline \multicolumn{10}{|c|}{ Sample (see notes) } \\
\hline & 1 & 2 & 3 & 4 & 5 & 6 & 7 & 8 & 9 \\
\hline $\mathrm{SiO}_{2}$ & $49.10 \%$ & $53.59 \%$ & $52.85 \%$ & $52.77 \%$ & $52.36 \%$ & $51.66 \%$ & $50.92 \%$ & $53.19 \%$ & $49.50 \%$ \\
\hline $\mathrm{TiO}_{2}$ & 0.61 & 0.32 & 0.32 & 0.36 & 0.52 & 0.48 & 0.55 & 0.38 & 0.90 \\
\hline $\mathrm{Al}_{2} \mathrm{O}_{3}$ & 7.21 & 3.07 & 2.75 & 3.13 & 3.93 & 1.95 & 6.56 & 2.89 & 3.22 \\
\hline $\mathrm{Cr}_{2} \mathrm{O}_{3}$ & 0.33 & 0.31 & 0.24 & 0.30 & 0.11 & 0.07 & 0.16 & 0.11 & 0.11 \\
\hline $\mathrm{FeO}$ & 8.20 & 6.13 & 6.14 & 5.84 & 8.04 & 19.63 & 7.97 & 10.17 & 14.67 \\
\hline $\mathrm{MnO}$ & 0.30 & 0.27 & 0.26 & 0.23 & 0.26 & 0.57 & 0.20 & 0.30 & 0.32 \\
\hline $\mathrm{MgO}$ & 14.29 & 17.00 & 17.78 & 17.02 & 15.54 & 16.54 & 15.77 & 20.69 & 11.40 \\
\hline $\mathrm{CaO}$ & 19.59 & 20.80 & 20.46 & 20.44 & 19.92 & 7.82 & 19.31 & 12.94 & 18.25 \\
\hline $\mathrm{Na}_{2} \mathrm{O}$ & 0.29 & 0.26 & 0.27 & 0.28 & 0.28 & 0.15 & 0.31 & 0.37 & 0.43 \\
\hline Total & $99.91 \%$ & $101.74 \%$ & $101.07 \%$ & $100.37 \%$ & $100.96 \%$ & $98.86 \%$ & $101.75 \%$ & $101.03 \%$ & $98.82 \%$ \\
\hline \multicolumn{10}{|c|}{ Atoms per 6 Oxygens } \\
\hline $\mathrm{Si}_{i \mathrm{i}}$ & 1.819 & 1.929 & 1.918 & 1.924 & 1.912 & 1.966 & 1.843 & 1.922 & 1.908 \\
\hline $\mathrm{Al}^{\mathrm{iv}}$ & 0.181 & 0.071 & 0.082 & 0.076 & 0.088 & 0.034 & 0.157 & 0.078 & 0.092 \\
\hline $\mathrm{Al}^{\mathrm{VI}}$ & 0.134 & 0.059 & 0.035 & 0.059 & 0.081 & 0.054 & 0.123 & 0.046 & 0.054 \\
\hline $\mathrm{Ti}$ & 0.017 & 0.009 & 0.009 & 0.010 & 0.014 & 0.014 & 0.015 & 0.010 & 0.026 \\
\hline $\mathrm{Cr}$ & 0.010 & 0.009 & 0.007 & 0.009 & 0.003 & 0.002 & 0.005 & 0.003 & 0.003 \\
\hline $\mathrm{Fe}$ & 0.254 & 0.184 & 0.186 & 0.178 & 0.245 & 0.625 & 0.241 & 0.307 & 0.473 \\
\hline Mn & 0.009 & 0.008 & 0.008 & 0.007 & 0.008 & 0.018 & 0.006 & 0.009 & 0.011 \\
\hline $\mathrm{Mg}$ & 0.789 & 0.912 & 0.962 & 0.925 & 0.846 & 0.938 & 0.850 & 1.115 & 0.655 \\
\hline $\mathrm{Ca}$ & 0.778 & 0.802 & 0.795 & 0.798 & 0.779 & 0.319 & 0.749 & 0.501 & 0.753 \\
\hline $\mathrm{Na}$ & 0.021 & 0.018 & 0.019 & 0.020 & 0.020 & 0.011 & 0.022 & 0.026 & 0.032 \\
\hline \multirow{5}{*}{\multicolumn{5}{|c|}{$\begin{array}{l}\text { Notes: } \\
\text { 1. Sample } 462 \mathrm{~A}-40-1,56-59 \mathrm{~cm} \text {, augite } 1 \text { rim } \\
\text { 2. Sample } 463 \mathrm{~A}-40-1,56-59 \mathrm{~cm} \text {, augite } 1 \text { core } \\
\text { 3. Sample } 462 \mathrm{~A}-40-1,56-59 \mathrm{~cm} \text {, augite } 2 \\
\text { 4. Sample } 462 \mathrm{~A}-40-1,56-59 \mathrm{~cm} \text {, endiopside } 4\end{array}$}} & \multicolumn{5}{|c|}{ 5. Sample $462 \mathrm{~A}-56-1,131-134 \mathrm{~cm}$, augite 1} \\
\hline & & & & & \multicolumn{5}{|c|}{ 6. Sample $462 \mathrm{~A}-56-1,131-134 \mathrm{~cm}$, subcalcic augite 2} \\
\hline & & & & & \multicolumn{5}{|c|}{ 7. Sample $462 \mathrm{~A}-56-1,131-134 \mathrm{~cm}$, augite 3} \\
\hline & & & & & \multicolumn{5}{|c|}{ 8. Sample $462 \mathrm{~A}-56-1,131-134 \mathrm{~cm}$, augite 5} \\
\hline & & & & & \multicolumn{5}{|c|}{ 9. Sample $462 \mathrm{~A}-56-1,131-134 \mathrm{~cm}$, augite 6} \\
\hline
\end{tabular}


Table 3. Plagioclase analyses.

\begin{tabular}{|c|c|c|c|c|c|c|c|c|c|c|c|c|c|c|}
\hline \multicolumn{15}{|c|}{ Sample (see notes) } \\
\hline & 1 & 2 & 3 & 4 & 5 & 6 & 7 & 8 & 9 & 10 & 11 & 12 & 13 & 14 \\
\hline $\mathrm{SiO}_{2}$ & $49.86 \%$ & $50.16 \%$ & $50.31 \%$ & $47.70 \%$ & $49.04 \%$ & $49.08 \%$ & $52.39 \%$ & $51.07 \%$ & $50.61 \%$ & $50.28 \%$ & $56.65 \%$ & $54.96 \%$ & $59.45 \%$ & $56.00 \%$ \\
\hline $\mathrm{Al}_{2} \mathrm{O}_{3}$ & 30.75 & 30.28 & 30.63 & 32.76 & 32.23 & 31.05 & 29.44 & 30.17 & 31.05 & 31.17 & 27.19 & 28.44 & 25.27 & 28.22 \\
\hline $\mathrm{CaO}$ & 15.13 & 15.03 & 14.85 & 16.87 & 15.97 & 15.59 & 12.93 & 14.43 & 15.03 & 15.45 & 10.00 & 11.53 & 7.74 & 10.86 \\
\hline $\mathrm{Na}_{2} \mathrm{O}$ & 3.02 & 3.07 & 3.04 & 2.09 & 2.36 & 2.74 & 3.91 & 3.34 & 3.05 & 2.82 & 5.92 & 5.00 & 7.06 & 5.27 \\
\hline $\mathrm{K}_{2} \mathrm{O}$ & 0.06 & 0.08 & 0.11 & 0.07 & 0.05 & 0.08 & 0.05 & 0.08 & 0.02 & 0.05 & 0.08 & 0.08 & 0.12 & 0.07 \\
\hline Total & $98.81 \%$ & $98.62 \%$ & $98.94 \%$ & $99.49 \%$ & $99.65 \%$ & $98.53 \%$ & $98.71 \%$ & $99.09 \%$ & $99.75 \%$ & $99.78 \%$ & $99.84 \%$ & $100.01 \%$ & $99.64 \%$ & $100.42 \%$ \\
\hline \multicolumn{15}{|c|}{ Atoms per 32 Oxygens } \\
\hline $\mathrm{Si}$ & 9.209 & 9.280 & 9.268 & 8.800 & 8.994 & 9.107 & 9.607 & 9.380 & 9.245 & 9.195 & 10.192 & 9.911 & 10.640 & 10.029 \\
\hline $\mathrm{Al}$ & 6.694 & 6.601 & 6.651 & 7.122 & 6.966 & 6.791 & 6.364 & 6.531 & 6.684 & 6.717 & 5.765 & 6.044 & 5.331 & 5.957 \\
\hline $\mathrm{Ca}$ & 2.993 & 2.979 & 2.931 & 3.335 & 3.138 & 3.098 & 2.540 & 2.840 & 2.941 & 3.027 & 1.927 & 2.228 & 1.484 & 2.083 \\
\hline $\mathrm{Na}$ & 1.080 & 1.101 & 1.087 & 0.747 & 0.839 & 0.984 & 1.389 & 1.189 & 1.080 & 1.001 & 2.065 & 1.747 & 2.451 & 1.830 \\
\hline K & 0.015 & 0.019 & 0.025 & 0.015 & 0.012 & 0.019 & 0.011 & 0.018 & 0.005 & 0.011 & 0.018 & 0.019 & 0.027 & 0.016 \\
\hline
\end{tabular}

Notes:

1. Sample 462A-40-1, 56-59 cm, phenocryst \#1, An73

2. Sample 462A-40-1, 56-59 cm, groundmass lath \#1, An73

3. Sample $462 A-40-1,56-59 \mathrm{~cm}$, phenocryst $\# 2$ core, An 72

4. Sample 462A-40-1, 56-59 cm, phenocryst \#2 rim, An 81

5. Sample 462A-40-1, 56-59 cm, phenocryst \#3 rim, An79

6. Sample 462A-40-1, 56-59 cm, phenocryst \#3 core, An76

7. Sample 462A-56-1,131-134 cm, phenocryst \#1 rim, An 64
8. Sample 462A-56-1, 131-134 cm, phenocryst \#1 core, An70

9. Sample 462A-56-1, 131-134 cm, phenocryst \#2 rim, An73

10. Sample 462A-56-1, 131-134 cm, phenocryst \#2 core, An75

11. Sample 462-69-1, 20-24 cm, phenocryst \#1 rim, An48

12. Sample 462-69-1, 20-24 cm, phenocryst \#1 core, Ans6

13. Sample $462-69-1,20-24 \mathrm{~cm}$, phenocryst \#2 rim, An38

14. Sample 462-69-1, 20-24 cm, phenocryst \#2 core, An 53
Table 4. $\delta^{18} \mathrm{O}(\mathrm{SMOW})$ analyses of Leg 61 basalts.

\begin{tabular}{lrll}
\hline \multicolumn{1}{c}{$\begin{array}{c}\text { Core-Sec., } \\
\text { Interval (cm) }\end{array}$} & $\delta^{18} \mathrm{O}(\%)$ & $\begin{array}{l}\text { Core-Sec., } \\
\text { Interval (cm) }\end{array}$ & $\delta^{18} \mathrm{O}(\%)$ \\
\hline Hole 462A & & $50-3,130-134$ & 7.4 \\
$14-2,82-87$ & 8.6 & $51-4,17-22$ & 6.7 \\
$15-166-68$ & 6.9 & $56-1,61-64$ & 6.5 \\
$17-2,84-86$ & 6.9 & $56-1,131-134$ & 6.0 \\
$20-1,116-121$ & 7.1 & $60-3,100-102$ & 6.6 \\
$21-1,140-143$ & 9.7 & $65-1,59-61$ & 6.3 \\
$22-2,120-129$ & 10.2 & $68-3,119-122$ & 6.5 \\
$27-1,94-97$ & 6.2 & $59-1,80-84$ & 6.1 \\
$28-3,1-2$ & 6.5 & $69-2,68-72$ & 8.0 \\
$29-3,78-82$ & 6.6 & $72-2,63-66$ & 6.3 \\
$32-1,24-28$ & 7.0 & $72-3,21-23$ & 6.5 \\
$38-2,53-56$ & 6.9 & $74-4,25-31$ & 7.2 \\
$39-2,100-104$ & 6.3 & $74-4,40-50$ & 7.5 \\
$41-4,86-90$ & 10.1 & $87-1,105-107$ & 6.7 \\
$44-1,66-71$ (gray) & 5.8 & $88-2,95-97$ & 6.1 \\
$44-1,66-71$ (brown) & 9.8 & $88-2,100-102$ & 8.8 \\
$47-2,90-94$ & 6.8 & $88-3,9-11$ & 8.6 \\
$49-3,65-68$ & 6.3 & $89-3,125-127$ & 6.3 \\
$50-3,11-14$ & 6.6 & $90-4,63-65$ & 7.3 \\
\hline
\end{tabular}

shortly after eruption near mid-ocean ridges (Scheidegger and Corliss, 1980). This similarity of alteration conditions is particularly interesting because it is known that the Nauru Basin igneous complex erupted on oceanic crust that was 30 to $50 \mathrm{~m}$.y. old, and not at an oceanic ridge crest (Batiza et al., 1980). This type of alteration thus seems to be independent of the tectonic setting of eruption, but it is controlled largely by direct exposure of the basalt to sea water.

After rapid eruption of the flows and a period of sedimentation, sills intruded into the overlying sedimentary section. The sills were probably altered by less oxidizing sedimentary pore fluids of high $P_{\mathrm{CO}_{2}}$, by a process similar to the one described by Einsele et al. (1980). Alteration minerals include smectite, calcite, pyrite, and quartz-a suite of secondary minerals common in MORB samples formed at mid-ocean ridges. In Leg 61 sills there is also abundant evidence of late magmatic alteration that preceded the non-oxidative alteration episode and resulted in the formation of granophyric patches and two generations of amphibole (Plate 2).

Superimposed on the results of previous episodes of alteration is evidence of an oxidative alteration episode that resulted in the stabilization of celadonite and $\mathrm{Fe}$ oxides and the destruction of other smectites and pyrite. These effects are mainly restricted to the upper portions of the complex, and provide evidence for rapid accumulation of the Nauru Basin igneous complex.

\section{Whole-Rock $\delta^{18} \mathrm{O}$ Analyses}

The $\delta^{18} \mathrm{O}$ values of 31 whole-rock powders, which were also used in some of the other studies, range from 5.8 to $10.2 \%$ SMOW (Standard Mean Ocean Water) (Table 4). The observed variation is similar to that found for previously described DSDP sites, but the average $\delta^{18} \mathrm{O}$ value, $\sim 7.2 \%$, is less than that found for the older holes (Muehlenbachs, 1980). No systematic difference of ${ }^{18} \mathrm{O}$ content between the top sills and bottom flows is evident. $\delta^{18} \mathrm{O}$-versus- $\mathrm{H}_{2} \mathrm{O}^{+}$systematics of four samples are similar to those of dredged and drilled basalts (Muehlenbachs and Clayton, 1972; Muehlenbachs, 1980), implying that the $\delta^{18} \mathrm{O}$ variation of the Hole 462A samples results from low-temperature alteration. The unaltered basalt has a $\delta^{18} \mathrm{O}$ of $5.8 \%$-identical to fresh MORB - but most samples are enriched in ${ }^{18} \mathrm{O}$ because of about $5 \%$ alteration, and a few are up to $20 \%$ altered. These most altered basalts $\left(\delta^{18} \mathrm{O} \sim 10 \%\right.$ ) were sampled near flow or sill margins, zones of high transmissivity of sea water.

The preferential low-temperature alteration of such zones was most obvious in material collected on Legs 51,52 , and 53 (Muehlenbachs, 1980), where much totally altered material was recovered $\left(\delta^{18} \mathrm{O}=18-26 \%\right)$. 
There is no obvious explanation why the Hole $462 \mathrm{~A}$ samples are less altered (less ${ }^{18} \mathrm{O}$ enriched) than the 100 -m.y.-old Hole 417A and Hole 418A basalts and other Cretaceous basalts. Sampling bias could explain this difference, in that some highly altered samples were specifically chosen from Holes 417A and 418A, whereas the least altered samples were selected from Hole $462 \mathrm{~A}$. The freshest massive basalts from the Atlantic holes have about the same $\delta^{18} \mathrm{O}$ as those from Hole $462 \mathrm{~A}$, but basalts near the top of the section and the already mentioned transmissive zones are much more altered. Perhaps the off-axis emplacement of the Hole $462 \mathrm{~A}$ rocks did not provide either as extensive a network of channels for sea water to penetrate or enough thermal energy to drive the convective system.

\section{Whole-Rock Chemical Analyses}

\section{Major Elements}

Onboard XRF analyses for $\mathrm{Si}, \mathrm{Al}, \mathrm{Ti}$, total $\mathrm{Fe}, \mathrm{Mn}$, $\mathrm{Mg}, \mathrm{Ca}, \mathrm{K}$, and $\mathrm{P}$ were completed for 33 samples, and for all these elements except $\mathrm{Mn}$ and $\mathrm{P}$ for another 113 samples of Leg 61 basalt. Additional wet-chemical analyses (42 total) and electron microprobe glass analyses are given in Tokuyama and Batiza (this volume), Shcheka (this volume). Analyses for $\mathrm{Al}, \mathrm{Ca}$, $\mathrm{Fe}, \mathrm{K}, \mathrm{Mg}, \mathrm{Mn}, \mathrm{Na}, \mathrm{P}$, and $\mathrm{Ti}$ for 15 additional samples are given by Fujii et al. (this volume), and majorelement analyses of 14 other samples are given by Seifert (this volume). The great similarity of results obtained by various methods in several laboratories indicates that no serious interlaboratory biases exist.

The sills are systematically more fractionated than the lower flow units. Sills have consistently more abundant $\mathrm{Ti}, \mathrm{Fe}$, and $\mathrm{K}$, and less abundant $\mathrm{Mg}$ and $\mathrm{Ca}$ than flows, with essentially no overlap in compositional range. It is very unlikely that these chemical differences result from oxidative alteration, because this alteration does not greatly affect rocks below Core $462 \mathrm{~A}-19$. Further, $\mathrm{Fe}^{+3} /$ to al $\mathrm{Fe}$ ratios of both sills and flows are relatively low: the mean is about 0.29 for the sills and about 0.36 for the flows, albeit with much scatter. Oxygen isotope data and petrographic observations also indicate that all the rocks are only slightly altered and that no large systematic differences exist in the degree of alteration. Thus, the systematic variation in chemical composition between the sills and the flows probably reflects differences between igneous petrogenetic processes in the two rock types.

Both sills and flows are chemically similar, but not identical to suites of MORB. Nauru Basin basalts are either quartz- or olivine-hypersthene-normative. The trends of chemical variation in the Nauru Basin suite are different in detail, however, from trends in fractionated suites of MORB. For example, at comparable $\mathrm{Mg} /(\mathrm{Mg}$ $+\mathrm{Fe}^{+2}$ ) ratios, the Nauru Basin basalts have systematically less $\mathrm{TiO}_{2}$ than suites of MORB (Shcheka, this volume; Tokuyama and Batiza, this volume; Batiza et al., 1980).

Systematic variation of chemical composition with stratigraphic position is apparent within the Nauru
Basin (Shcheka, this volume; Batiza, this volume). In the upper sill unit, the degree of enrichment in $\mathrm{Ti}$ and $\mathrm{Fe}$ generally correlates with stratigraphic position: $\mathrm{Ti}$ - and Fe-rich rocks are stratigraphically higher in the section, although there is some scatter. In contrast, the flow section shows the opposite relationship: flows higher in the section are systematically more depleted in $\mathrm{Fe}$ and $\mathrm{Ti}$ than those near the base. There is essentially no compositional overlap between the flow section and the sill section, although both are chemically similar.

This variation is reflected in systematically higher $\mathrm{Mg} /\left(\mathrm{Mg}+\mathrm{Fe}^{+2}\right)$ for flows, compared with sills. Most of the flows have $\mathrm{Mg} /\left(\mathrm{Mg}+\mathrm{Fe}^{+2}\right)>0.60$, and many are about $0.64-0.65$, indicating that they may be primary mantle melts that have undergone relatively little fractionation.

\section{Trace Elements}

Batiza (this volume) and Seifert (this volume) give instrumental neutron-activation analysis (INAA) determinations of seven rare-earth elements (REE) and $\mathrm{FeO}$, $\mathrm{Sc}, \mathrm{Cr}, \mathrm{Co}, \mathrm{Ni}, \mathrm{Na}_{2} \mathrm{O}, \mathrm{Hf}$, and $\mathrm{Th}$ for 52 sample sof $\mathrm{Leg}$ 61 basalt. Shcheka (this volume), citing results for 30 samples, gives analyses of $\mathrm{Zr}, \mathrm{Rb}$, and $\mathrm{Sr}$ by $\mathrm{XRF}$ methods, $\mathrm{Cr}, \mathrm{Ni}, \mathrm{Co}$, and $\mathrm{Zn}$, by atomic absorption methods, and $\mathrm{V}$ and $\mathrm{Sn}$ by spectroscopic methods. Fujii et al. (this volume) present analyses of $\mathrm{Ba}, \mathrm{Li}, \mathrm{Sc}, \mathrm{Sr}, \mathrm{V}$, and Y by optical emission spectrometric methods for 17 samples and $\mathrm{Sr}$ and $\mathrm{Rb}$ analyses by isotope dilution for 3 samples. Tokuyama and Batiza (this volume) present additional $\mathrm{Ni}, \mathrm{Co}, \mathrm{Cr}$, and $\mathrm{S}$ atomic absorption analyses for 7 samples. Considering the analytical precision and accuracy of the various methods used, these results are in excellent agreement.

Findings based on trace-element data agree with those from major-element data in the following: (1) the basalts of the Nauru Basin igneous complex are similar, but not identical to MORB; (2) the sills are more fractionated than the flows; (3) systematic stratigraphic compositional trends exist within both the sills and flows; and (4) the chemical effects of alteration are minimal. Observed trace-element variations reflect primary igneous processes and not the chemical effects of alteration. Some observed scatter in the chemical variation of some major and trace elements may, however, be attributable to the effects of alteration.

Both sills and flows show depletion of the light REE relative to the heavy REE, as do MORB samples. The flows have $\mathrm{K} / \mathrm{Rb}=1050-1100, \mathrm{~K} / \mathrm{Ba}>50, \mathrm{Rb}<1$ $\mathrm{ppm}, \mathrm{Sr}=\sim 100 \mathrm{ppm}, \mathrm{Zr}=\sim 80 \mathrm{ppm}$, and other similarities to MORB with respect to trace elements. The sills contain slightly higher levels of incompatible trace elements, but ratios of these elements are similar to ratios in the flows. This is consistent with greater fractionation of the sills.

\section{Isotopic Data}

Fujii et al. (this volume) give ${ }^{87} \mathrm{Sr} / 86 \mathrm{Sr}$ data for 17 whole-rock basalt samples of Leg 61 basalt. One sample was leached in $6 \mathrm{~N} \mathrm{HCl}$ at $110^{\circ} \mathrm{C}$ overnight and showed no change of ${ }^{87} \mathrm{Sr} / 86 \mathrm{Sr}$. Nauru Basin basalts have 
${ }^{87} \mathrm{Sr} / 86 \mathrm{Sr}=0.70370 \pm 0.00011$, which is significantly higher than normal MORB. Fujii et al. (this volume) point out that sea water contamination or some other process could be responsible for this difference. These ratios are not, of course, initial ${ }^{87} \mathrm{Sr} /{ }^{86} \mathrm{Sr}$ ratios, but the $\mathrm{Rb} / \mathrm{Sr}$ of the rocks is so low that even 200 m.y. of growth in the $\mathrm{Rb}-\mathrm{Sr}$ system could not account entirely for the observed difference between Nauru Basin basalt and normal MORB.

\section{SUMMARY AND CONCLUSIONS}

The Nauru Basin igneous complex consists of two major units: an upper unit of sills and a lower extrusive unit of sheet-flows. Both of these basalt units were emplaced on oceanic crust 30 to 50 m.y. old, and thus represent Cretaceous off-ridge volcanic activity.

Basalts of the complex include many textural types and contain olivine (pseudomorphs and fresh crystals), spinel, augite, pigeonite, plagioclase, and $\mathrm{Fe}-\mathrm{Ti}$ oxides, as well as secondary and accessory phases. The chemical compositions of coexisting olivine, spinel, and basaltic glass are significantly different from MORB, but feldspar and pyroxene compositions are typical of MORB.

Major- and trace-element compositions of Nauru Basin igneous complex basalts are also similar to those of MORB, but the chemical trends of variation are significantly different from those of suites of MORB. The basalts are quartz and olivine-hypersthene normative tholeiites with rare-earth abundance patterns indicating depletion in the light REE. A variety of other traceelement abundances and trace-element ratios are similar to those observed in MORB.

Oxygen isotopic data indicate that the basalts are not as extensively altered as Layer 2 basalts of comparable age in the Atlantic. Studies of secondary minerals which occur in the Nauru Basin basalts indicate two alteration episodes at different times and under physical/chemical conditions. The chemical effects of alteration do not appear to be large, and so the orderly stratigraphicchemical relations in Hole $462 \mathrm{~A}$ samples are almost certainly primary.

It is possible that the Nauru Basin basalt complex is a part of, or is related to, the volcanism of the OntongJava plateau. If so, the complex is part of a very voluminous basalt outpouring chemically similar to the flood basalts of the Caribbean. This mode of oceanic volcanism may therefore be more common and volumetrically more important than has previously been thought.

\section{REFERENCES}

Ballard, R. D., Holcomb, R. T., and van Andel, Tj. H., 1979. The Galapagos rift at $86^{\circ} \mathrm{W}, 3$, sheet flows, collapse pits and lava lakes of the rift valley. J. Geophys. Res., 84:5407-5422.

Batiza, R., Larson, R. L., Schlanger, S. O., et al., 1980. Trace element abundances in basalts of Nauru Basin (DSDP Leg 61): Late Cretaceous off-ridge volcanism in the western Pacific. Nature, 286:476-478.

Einsele, G., Gieskes, G. M., Curray, J., et al., 1980. Intrusion of a basaltic sill into highly porous sediments, and resulting hydrothermal activity. Nature, 283:441-445.

Muehlenbachs, K., 1980. The alteration and aging of the basaltic layer of the sea floor: Oxygen isotope evidence from DSDP/IPOD Legs 51, 52, and 53. In Donnelly, T., Francheteau, J., Bryan, W., Robinson, P., Flower, M., Salisbury, M., et al., Init. Repts. $D S D P, 51,52,53$, Pt. 2: Washington (U.S. Govt. Printing Office), 1159-1167.

Muehlenbachs, K., and Clayton, R. N., 1972. Oxygen isotope studies of fresh weathered submarine basalts. Can. J. Earth Sci., 9:172184.

Roeder, P. L., Campbell, I. H., and Jamieson, H. E., 1979. A reevaluation of the olivine-spinel geothermometer. Contrib. Mineral. Petrol., 68:325-334.

Scheidegger, K. F., and Corliss, J. B., in press. Petrogenesis and secondary alteration of upper layer 2 basalts of the Nazca Plate. Mem.-Geol. Soc. Am. 


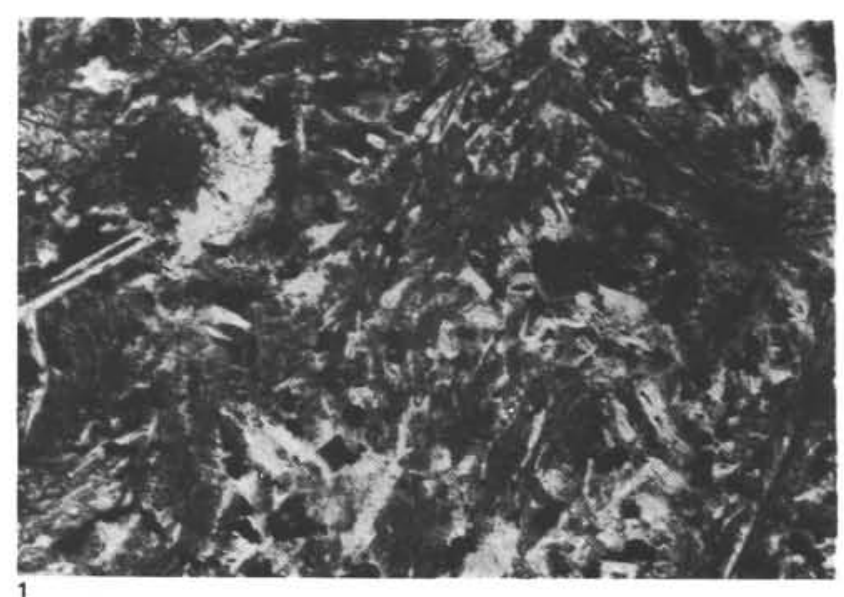

1

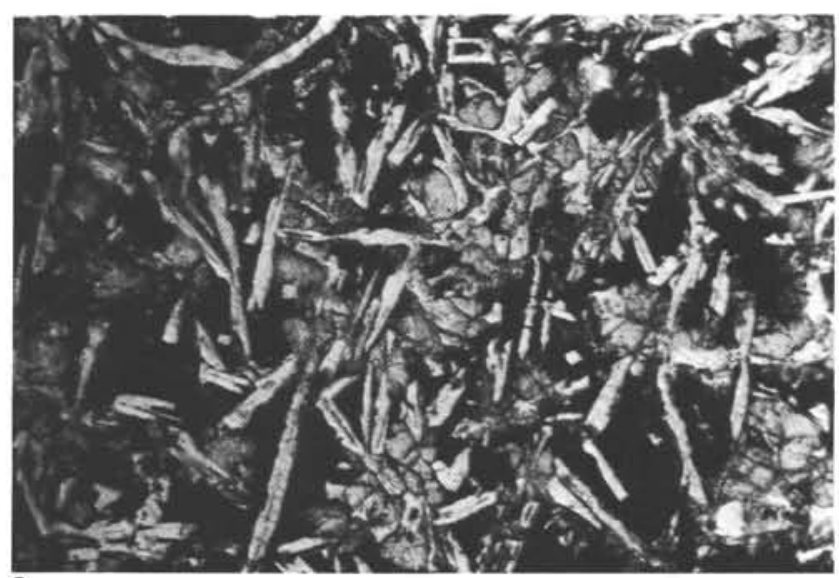

3

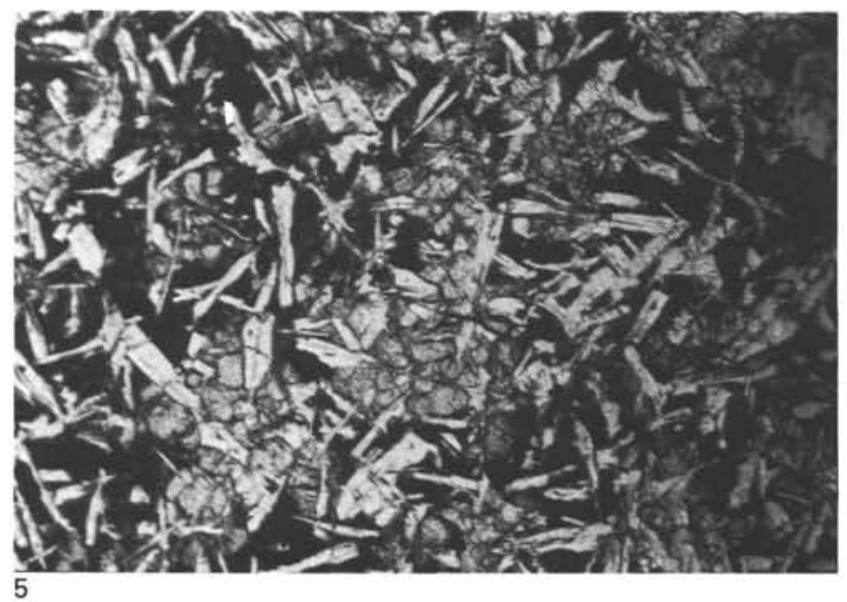

Plate 1. Thin sections.

Figure 1. Typical variolitic texture: Sample $462-60-2,53-57 \mathrm{~cm}$. (Field of view is $1.0 \mathrm{~mm}$.)

Figure 2. Typical hyalopilitic texture in Sample 462-60-2, 135-139 cm. (Field of view is $2.0 \mathrm{~mm}$.)

Figure 3. Typical intersertal to intergranular texture in Sample $462-61-1,105-108 \mathrm{~cm}$. (Field of view is $2.0 \mathrm{~mm}$.)
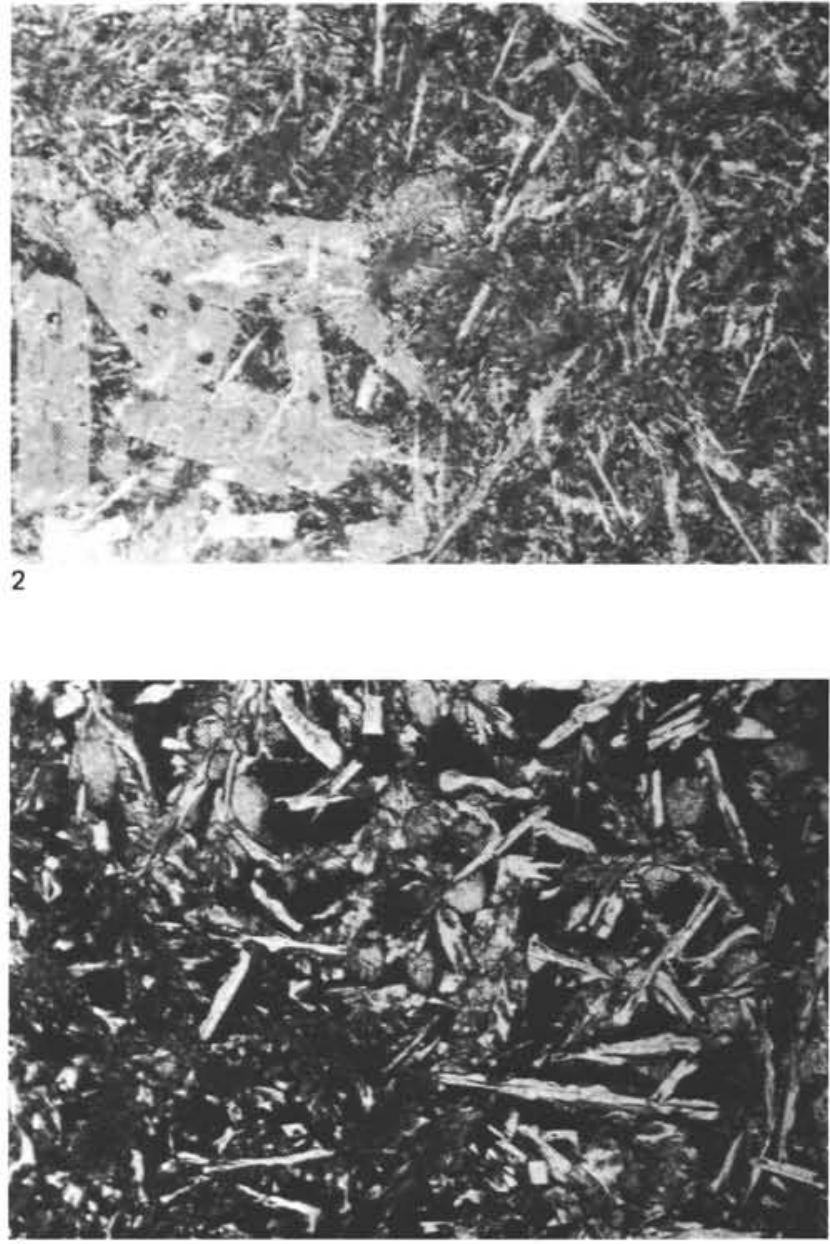

4

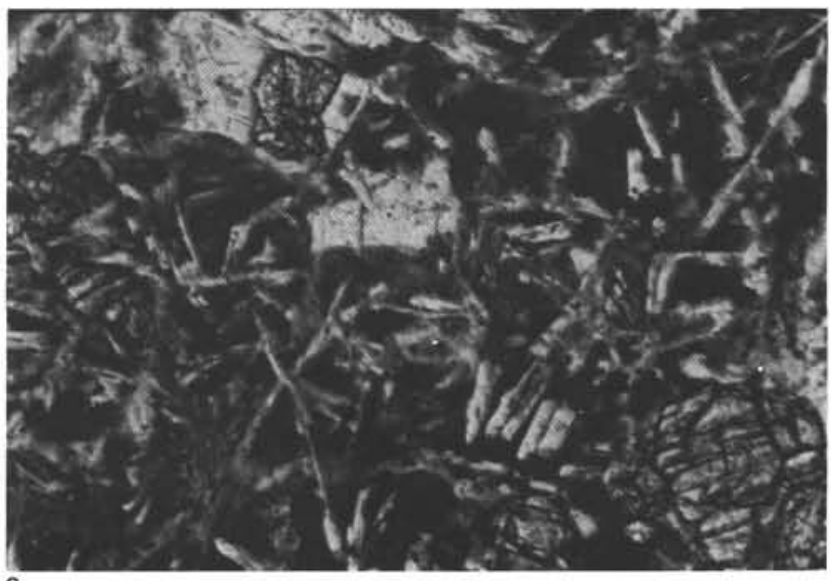

Figure 4. Predominantly intersertal to intergranular texture basalt with finer grained patch in lower left portion of photomicrograph: Sample $462-61-1,105-108 \mathrm{~cm}$. (Field of view is $2.0 \mathrm{~mm}$.)

Figure 5. Typical diabasic texture in Sample 462-61-2, 53-56 cm. (Field of view is $2.0 \mathrm{~mm}$.)

Figure 6. Hyalopilitic texture in Sample 462-63-1, 64-68 cm. (Field of view is $1.0 \mathrm{~mm}$.) 

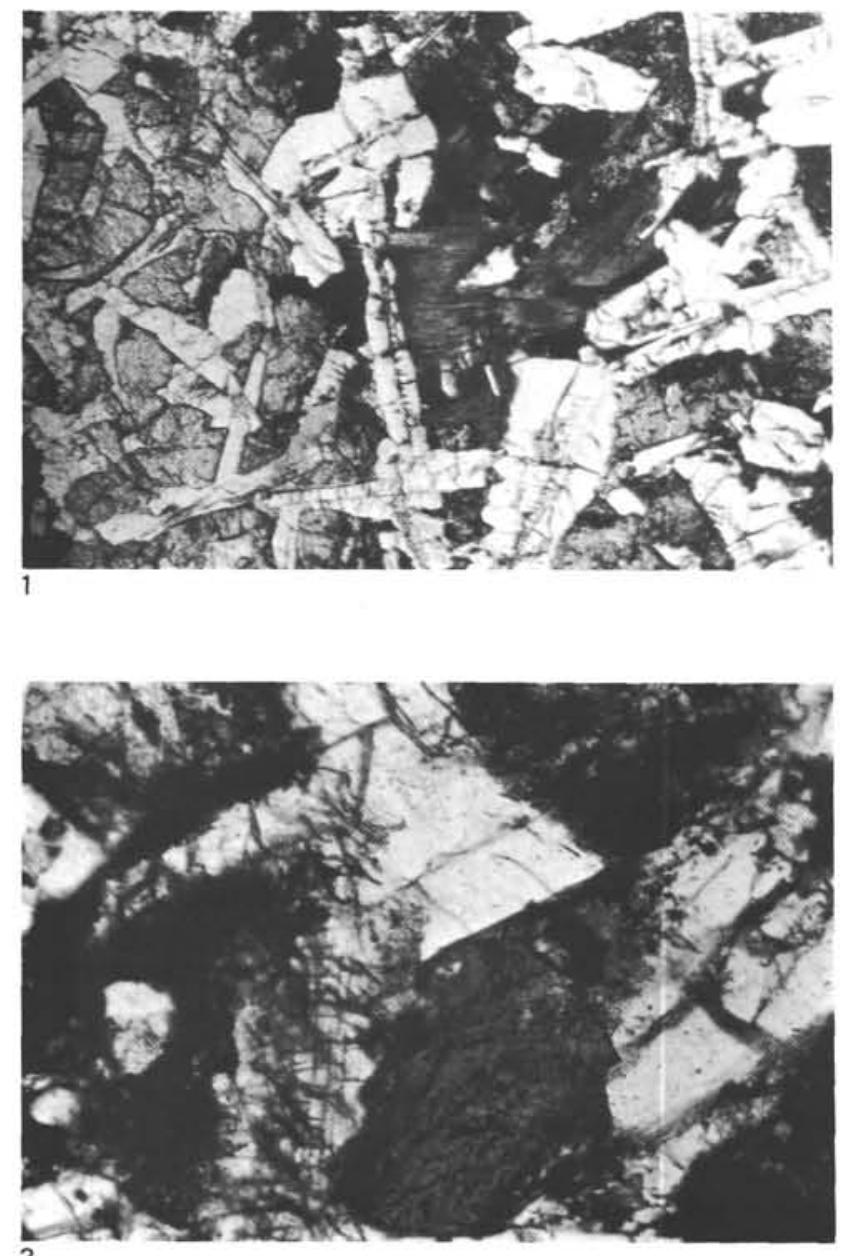

3

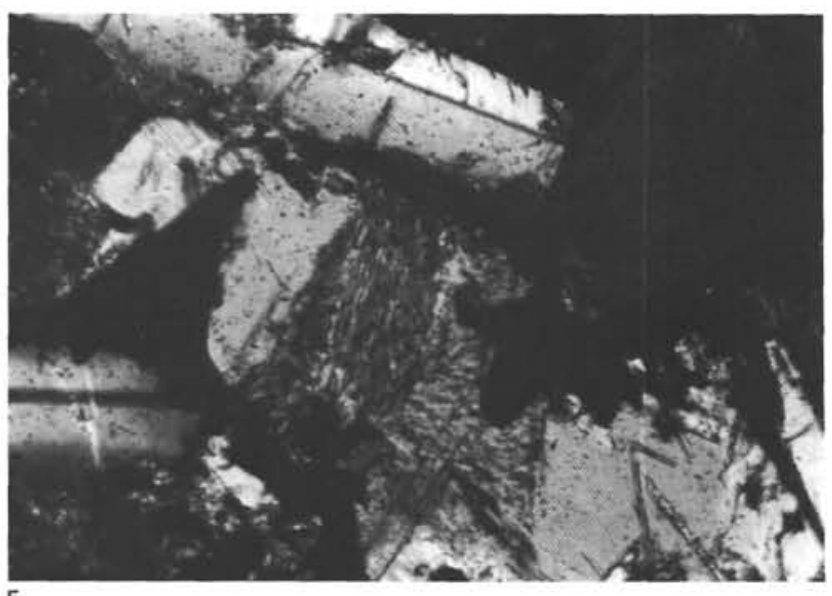

Plate 2. Thin sections.

Figure 1. Large amphibole crystal (dark) in Sample 462-68-1, 73-75 $\mathrm{cm}$. (Field of view is $2.0 \mathrm{~mm}$.)

Figure 2. Brown amphibole crystal (acicular and interstitial) in Sample $462-68-1,73-75 \mathrm{~cm}$. (Field of view is $1.0 \mathrm{~mm}$.)

Figure 3. Large grain of green amphibole (bottom center part of photomicrograph) in Sample 462-68-1, 73-75 cm. (Field of view is $1.0 \mathrm{~mm}$.
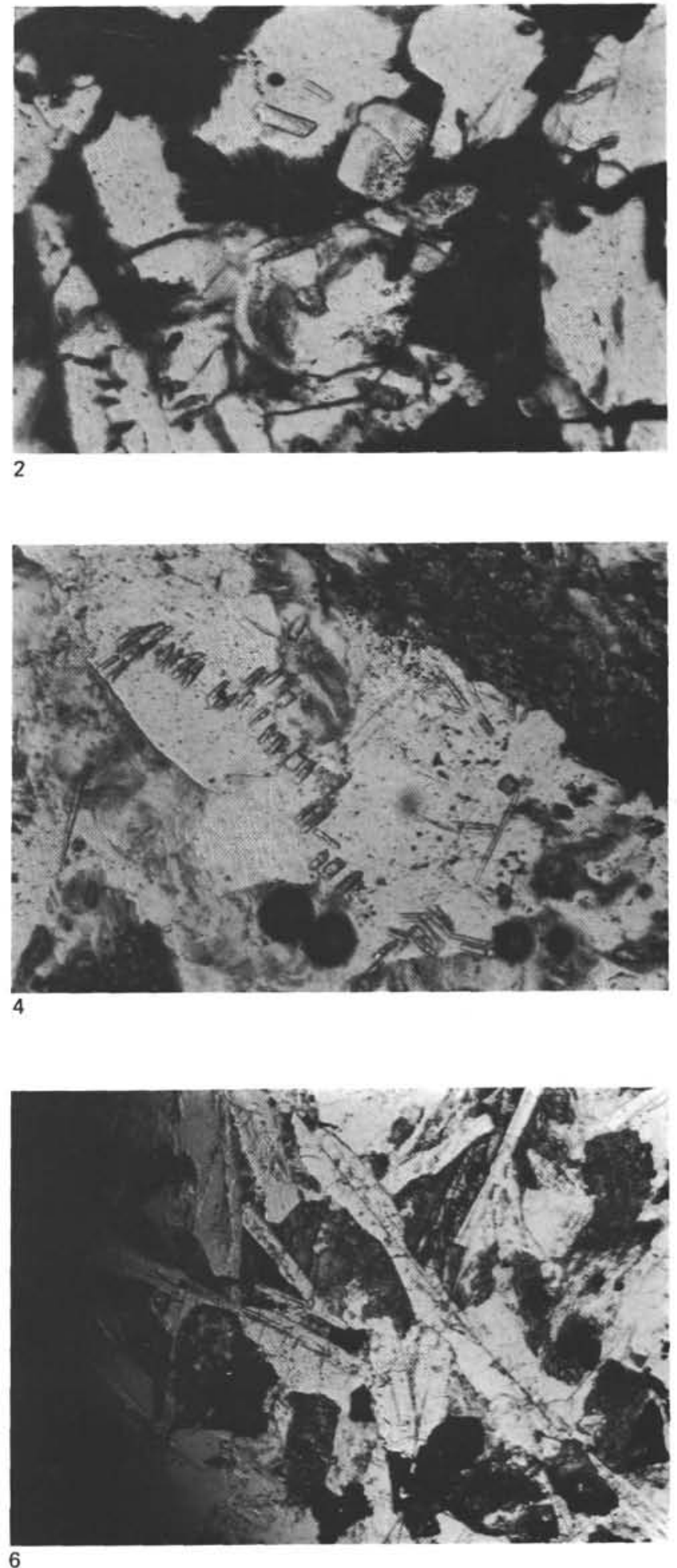

Figure 4. Large crystal of quartz with apatite inclusions in granophyric patch of Sample 462-69-1, 36-38 cm. (Field of view is 1.0 $\mathrm{mm}$.)

Figure 5. Central part of photomicrograph shows a quartz-potassium feldspar intergrowth bordered on two sides by quartz: Sample $462-66-2,65-67 \mathrm{~cm}$. (Field of view is $1.0 \mathrm{~mm}$.)

Figure 6. Very coarse grained texture of Sample 462-69-1, 36-38 cm. (Field of view is $4.0 \mathrm{~mm}$.) 


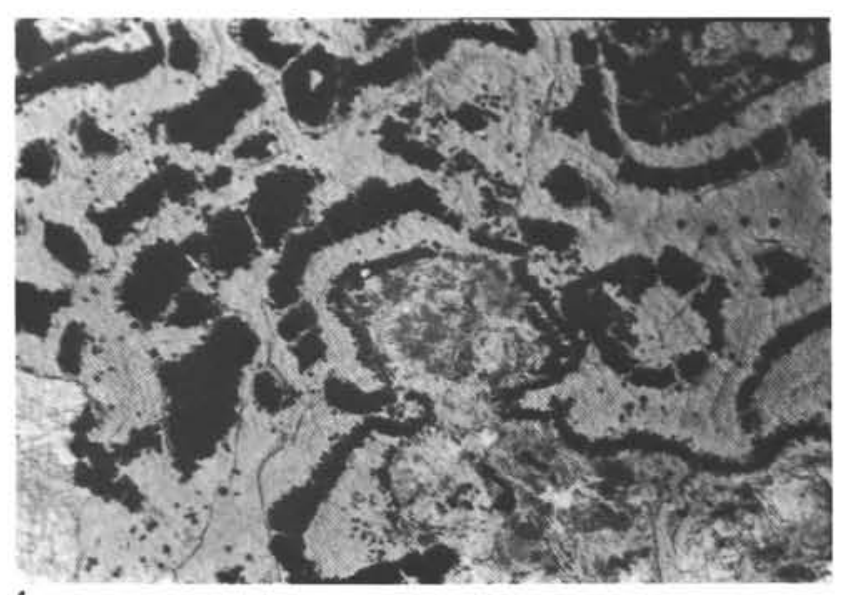

1

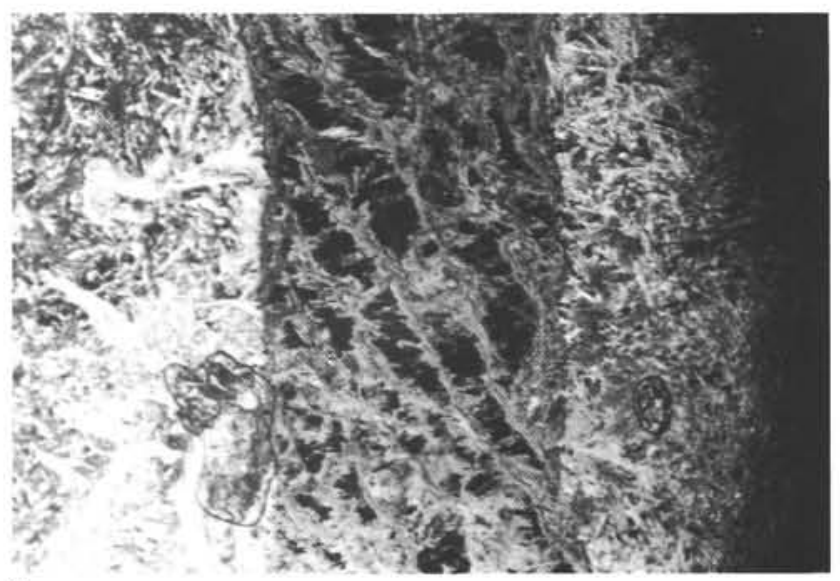

3

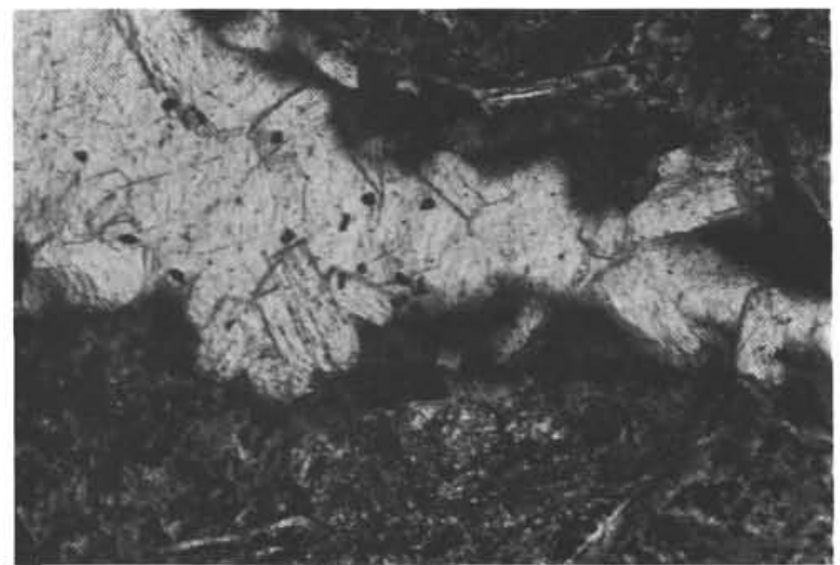

5

Plate 3. Thin sections.

Figure 1. View of secondary green smectite and iron-manganese oxides in Sample 462-60-2, 103-107 cm. (Field of view is $2.0 \mathrm{~mm}$.)

Figure 2. Part of a secondary-mineral vein in Sample 462-60-2, 53-57 $\mathrm{cm}$, showing two types of smectite and calcite. (Field of view is 1.0 $\mathrm{mm}$.)

Figure 3. Vein of smecite and opaque material in Sample 462-60-2, $103-107 \mathrm{~cm}$. (Field of view is $4.0 \mathrm{~mm}$.)
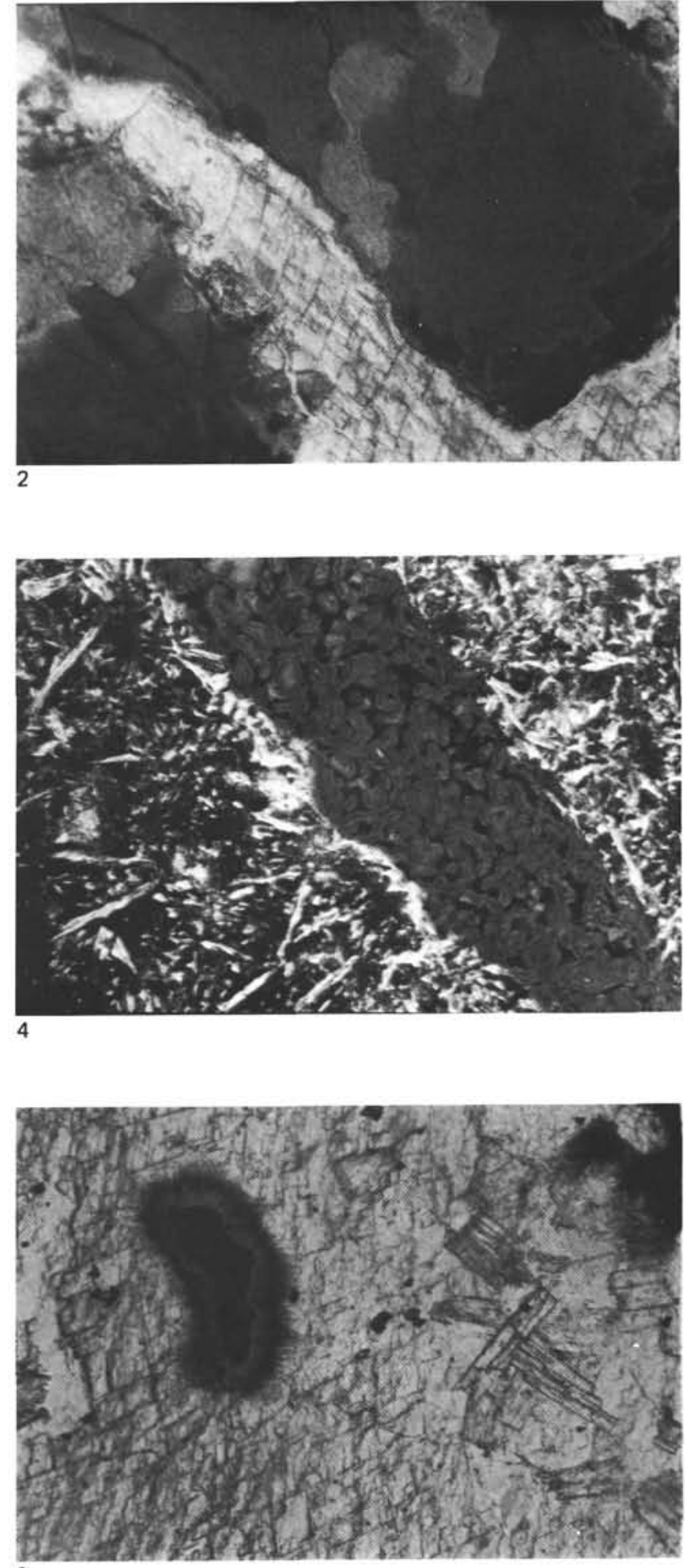

Figure 4. Vein of smecite and opaque material in Sample 462-60-2, $103-107 \mathrm{~cm}$. (Field of view is $4.0 \mathrm{~mm}$.)

Figure 5. Zoned secondary-mineral vein in Sample 462-60-1, 55-59 $\mathrm{cm}$. Outer portion of vein is celadonite (dark) and inner part is calcite. Both minerals are replaced by zeolite (euhedral). (Field of view is $3.0 \mathrm{~mm}$.)

Figure 6. Same vein as shown in Fig. 5. Dark patch consists of iron-manganese oxide with celadonite rim. Zeolite replaces calcite on the right side of photomicrograph. (Field of view is $1.0 \mathrm{~mm}$.) 

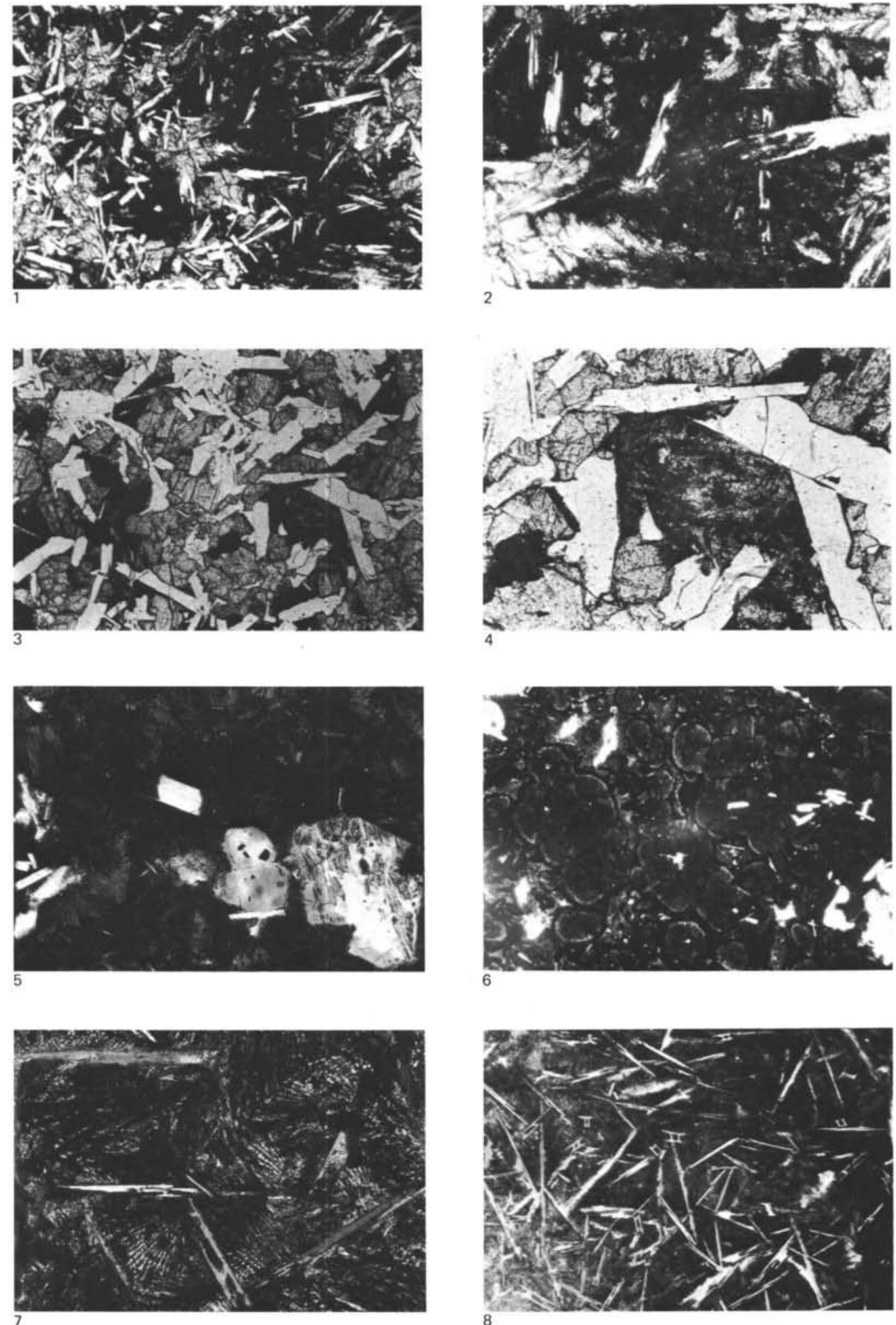

Plate 4. Thin sections.

Figure 1. Fine-grained patch in basalt (right side): Sample 462A-20-2, $93-95 \mathrm{~cm}$. (Field of view is $2.0 \mathrm{~mm}$.)

Figure 2. Close-up of fine-grained patch in Fig. 1. (Field of view is 1.0 $\mathrm{mm}$.)

igure 3. Interstitial hyalopilitic patch in basalt: Sample 462A-28-2, $28-30 \mathrm{~cm}$. (Field of view is $2.0 \mathrm{~mm}$.)

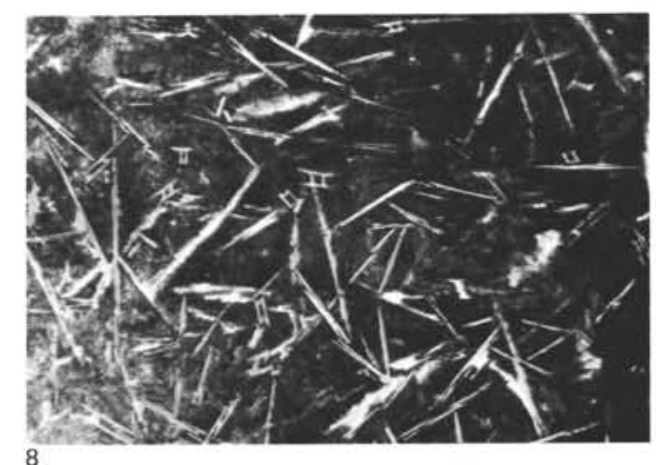

Figure 4. Close-up of Fig. 3. (Field of view is $1.0 \mathrm{~mm}$.)

Figure 5. Smetite after olivine (lower right) in Sample 462A-49-1, $55-57 \mathrm{~cm}$. (Field of view is $1.0 \mathrm{~mm}$.)

Figure 6. Spherulitic texture in altered glass in Sample 462A-49-1, $55-57 \mathrm{~cm}$. (Field of view is $2.0 \mathrm{~mm}$.)

Figure 7. Spinifex-like texture in Sample 462A-45-2, 122-124 cm. (Field of view is $1.0 \mathrm{~mm}$.)

Figure 8. Skeletal plagioclase crystals in Sample 462A-45-2, 14-17 cm. (Field of view is $2.0 \mathrm{~mm}$.) 